\title{
Current-driven switching of exchange biased spin-valve giant magnetoresistive nanopillars using a conducting nanoprobe
}

\author{
J. Hayakawa ${ }^{a)}$ and K. Ito \\ Hitachi, Ltd., Advanced Research Laboratory, 1-280, Higashi-koigakubo, Kokubunji-shi, \\ Tokyo 185-8601, Japan \\ Research Institute of Electrical Comunication, Tohoku University, 2-1-1 Katahira, Aobaku, \\ Sendai 980-0871, Japan \\ M. Fujimori, S. Heike, and T. Hashizume \\ Hitachi, Ltd., Advanced Research Laboratory, 2520, Hatoyama, Saitama 350-0395, Japan
}

J. Steen and J. Brugger

Microsystems Laboratory Ecole Polytechnique Federale de Lausanne (EPFL), CH-1015 Lausanne, Switzerland

\section{H. Ohno}

Research Institute of Electrical Communication, Tohoku University, 2-1-1 Katahira, Aobaku, Sendai 980-0871, Japan

(Received 17 February 2004; accepted 14 May 2004)

\begin{abstract}
An array of exchange biased spin-valve giant-magnetoresistance nanopillars was fabricated and the current $I$ dependence of the resistance $R$ was investigated using an electrically conducting atomic-force microscope (AFM) probe contact at room temperature. We observed current induced switching in a MnIr/CoFe $/ \mathrm{Cu} / \mathrm{CoFe} / \mathrm{NiFe}$ nanopillar using the $\mathrm{AFM}$ probe contact. Current-driven switching using nanoprobe contact is a powerful method for developing nonvolatile and rewritable magnetic memory with high density. () 2004 American Institute of Physics.
\end{abstract}

[DOI: $10.1063 / 1.1769605]$

\section{INTRODUCTION}

From both technological and fundamental viewpoints, the realization of future nonvolatile magnetic memory devices with higher density requires new approaches to reading and writing data because conventional magnetic data storage is rapidly approaching the limit of data writing. As the magnetic elements become smaller, a larger external magnetic field will be required to write magnetic data into them. Electrical switching by injection of a spin-polarized current, which was theoretically predicted by Slonczewski ${ }^{1}$ and Berger, ${ }^{2}$ has attracted attention because it would enable magnetic data to be written without an external magnetic field. In a $\mathrm{Co} / \mathrm{Cu} / \mathrm{Co}$ nanostructure, the hysteretic behavior of electrical switching when a current is applied has been experimentally demonstrated, ${ }^{3-10}$ the electrical switching is caused by the magnetic momentum reversal of the thinner Co layer due to the spin-polarized current injected from the thicker Co layer.

An alternative approach to ultrahigh density memory and data storage systems has been proposed ${ }^{11}$ in which data are written on a nonmagnetic polymer medium using a heating process and an atomic-force microscope AFM probe technique. Although reading and writing data electrically using proven magnetic technology it is more versatile and desirable, electrical magnetic recording using a probe contact in patterned magnetic elements has yet to be demonstrated. This paper reports the first observation of current-driven

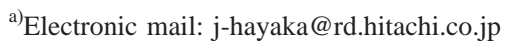

switching using a AFM nanoprobe contact on an exchange biased spin-valve giant magnetoresistance (GMR) nanopillar.

The nanopillar structure was an exchange biased spinvalve $\mathrm{Mn}_{80} \mathrm{Ir}_{20}(12 \mathrm{~nm}) / \mathrm{Co}_{90} \mathrm{Fe}_{10}(3 \mathrm{~nm}) / \mathrm{Cu}(6 \mathrm{~nm}) / \mathrm{Co}_{90} \mathrm{Fe}_{10}$ $(1 \mathrm{~nm}) / \mathrm{Ni}_{81} \mathrm{Fe}_{19}(5 \mathrm{~nm})[\mathrm{MnIr} / \mathrm{CoFe} / \mathrm{Cu} / \mathrm{CoFe} / \mathrm{NiFe}] \mathrm{GMR}$ film, in which the bottom $\mathrm{CoFe} / \mathrm{NiFe}$ acts as a magnetically soft layer, and the top CoFe layer acts as a magnetically hard layer. We selected this structure for two reasons.

(1) Using an array of a nanometer-scale pillars may enable the production of higher density magnetic memory device, although the shape of the pillars makes it difficult to control their magnetic anisotropy.

(2) Current-driven switching has been mainly observed in $\mathrm{Co} / \mathrm{Cu} / \mathrm{Co}$-based magnetic multilayered nanostructures $^{3-10}$ containing thick and thin Co layers. In previous experiments by others, ${ }^{6,10}$ an antiparallel configuration of the magnetization in two Co layers was produced using the difference in the coercive force $H_{c}$ of two Co ferromagnetic layers which is due to shape magnetic anisotropy. In our experiment, an exchange bias coupling using an antiferromagnet MnIr was employed to induce unidirectional magnetic anisotropy in the top layer to $\mathrm{CoFe}$ ensure either parallel or antiparallel magnetization of the $\mathrm{CoFe} / \mathrm{NiFe}$ and $\mathrm{CoFe}$ layers regardless of the pillar shape.

\section{EXPERIMENT}

We first prepared an $\mathrm{Au}(10 \mathrm{~nm}) / \mathrm{Mn}_{80} \mathrm{Ir}_{20}(12 \mathrm{~nm})$ $\mathrm{Co}_{90} \mathrm{Fe}_{10}(3 \mathrm{~nm}) / \mathrm{Cu}(6 \mathrm{~nm}) / \mathrm{Co}_{90} \mathrm{Fe}_{10}(1 \mathrm{~nm}) / \mathrm{Ni}_{81} \mathrm{Fe}_{19}(5 \mathrm{~nm}) /$ 

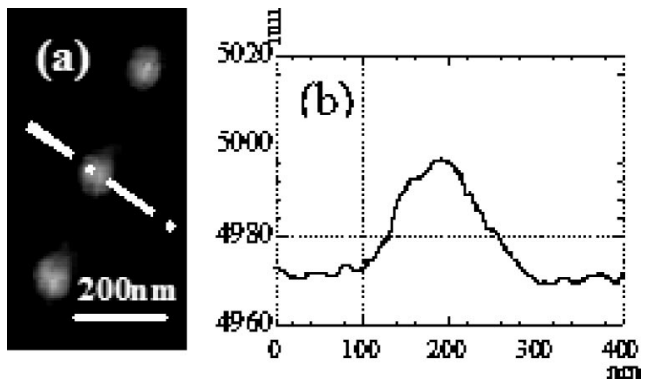

FIG. 1. (a) (SEM) image of an array of $\mathrm{MnIr} / \mathrm{CoFe} / \mathrm{Cu} / \mathrm{CoFe} / \mathrm{NiFe}$ nanopillars after ion milling; (b) AFM image of profile of a typical $\mathrm{MnIr} / \mathrm{CoFe} / \mathrm{Cu} / \mathrm{CoFe} / \mathrm{NiFe}$ pillar. Schematic view of cross section of nanopillar using an AFM apparatus.

$\mathrm{Ta}(50 \mathrm{~nm})$ film on a thermal-oxidized Si substrate using an ultrahigh vacuum sputtering apparatus. We fabricated the nanopillars using lithography with an AFM cantilever and ion milling. The milling step was timed to stop when the surface of the bottom NiFe layer appeared. The $\mathrm{MnIr} / \mathrm{CoFe} / \mathrm{Cu} / \mathrm{CoFe} / \mathrm{NiFe}$ nanopillars (100 nm diameter) formed in a uniform array with a $200 \mathrm{~nm}$ pitch. Figure 1(a) shows a scanning electron microscopy (SEM) image of an array of $\mathrm{MnIr} / \mathrm{CoFe} / \mathrm{Cu} / \mathrm{CoFe} / \mathrm{NiFe}$ nanopillars after ion milling. Figure 1(b) shows an AFM profile of a typical pillar; it shows that the pillar was $30 \mathrm{~nm}$ high and that the ion milling stopped when the surface of the bottom CoFe or $\mathrm{NiFe}$ layer appeared. Figure 2 shows our experimental setup. The dc current-voltage $(I-V)$ curve was measured using a conductive AFM (C-AFM) apparatus at room temperature, where the applied atomic force was from 5 to $10 \mathrm{nN}$. To establish ohmic contact between the probe and the pillar, we examined the $I-V$ characteristics of the Au film using four kinds of probes, Pt, PtIr, W, and B-doped C. The bias current direction was defined as positive when spin-polarized electrons flowed from the bottom $\mathrm{CoFe} / \mathrm{NiFe}$ layer to the top $\mathrm{CoFe}$ layer. The $I-V$ curve measurement was done under a zero magnetic field.

\section{RESULTS AND DISCUSSION}

Figures 3(a) and 3(b) show the $I-V$ curve for a contact on a separately prepared $50 \mathrm{~nm}$ Au film. The ones in (a) were measured using a Pt- and PtIr-coated Si cantilever while the ones in (b) were measured using B-doped C- and W-coated $\mathrm{Si}$ cantilevers. The curves in (a) showed a resistance of $\sim 100 \Omega$, while those in (b) exhibited diodelike characteris-

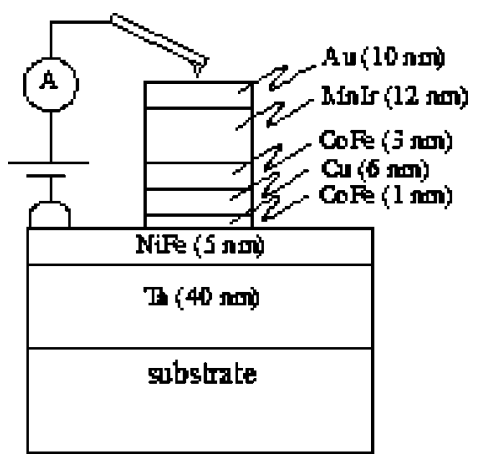

FIG. 2. Schematic view of cross section of experimental setup.
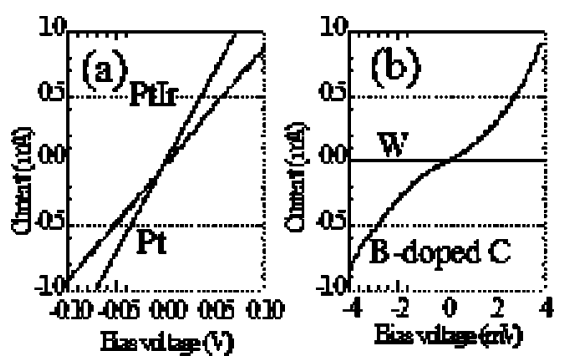

FIG. 3. (a) $I-V$ curves measured using Pt- and PtIr-coated Si cantilevers; (b) $I$ - $V$ curves measured using B-doped $\mathrm{C}$ - and $\mathrm{W}$-coated $\mathrm{Si}$ cantilever.

tics, suggesting that an oxidized layer had formed on the surface of the probe. A nonlinear $I-V$ curve like a tunnel contact was observed for the C-coated probe. The contact between the Au film and Pt or PtIr probe ensured an ohmic contact, which is why we used Au for both a protection cap and electrical contact for the nanopillars.

Figure 4 shows the typical $I$ dependence of $R$ in the $\mathrm{Au}(10 \mathrm{~nm}) / \mathrm{MnIr}(12 \mathrm{~nm}) / \mathrm{CoFe}(3 \mathrm{~nm}) / \mathrm{Cu}(6 \mathrm{~nm}) /$ $\mathrm{CoFe}(1 \mathrm{~nm}) / \mathrm{NiFe}(5 \mathrm{~nm})$ nanopillars when we used a Pt-coated probe. Clear hysteretic switching behavior was observed in these pillars with good reproducibility. We confirmed that no hysteretic behavior was present in an unpatterned GMR film. In the measurements in this study, when the current was flowing from the thinner top CoFe layer to the thicker bottom $\mathrm{CoFe} / \mathrm{NiFe}$ layer, the resistance changed from a higher state to a lower one. The observed $\Delta R / R$ was $2.7 \%$, which is in line with the spin-transfer torque-driven switching reported elesewhere ${ }^{5-10}$ for $\mathrm{Co} / \mathrm{Cu} / \mathrm{Co}$ nanopillars, as predicted by Slonczewski's model. ${ }^{1}$ The critical current values are $I c_{+} \sim 6.5 \mathrm{~mA}$ and $I c_{-} \sim-7 \mathrm{~mA}$ which correspond to $J c_{+}=8.3 \times 10^{7} \mathrm{~A} / \mathrm{cm}^{2}$ and $J c_{-}=8.9 \times 10^{7} \mathrm{~A} / \mathrm{cm}^{2}$, respectively.

Using the four kinds of probes, we examined the magnetic field $H$ dependence of $R$ for a different nanopillar having the same structure and dimensions. Figure 5 shows the $R$-Hcurves when the magnetic field was applied parallel to the direction of the unidirectional anisotropy of the top CoFe layer, as induced by the MnIr layer. The current was $1 \mu \mathrm{A}$; its geometry is shown in the inset. A typical $R-H$ loop of an exchange biased spin-valve GMR device was observed with $\Delta R / R$ of $2.5 \%$ between 0.1 and $0.25 \mathrm{kOe}$, which is in good agreement with the $R-I$ measurement using probe contact (Fig. 4). This result suggests that the electrical switching produced using a probe contact is caused by current-driven magnetization reversal.

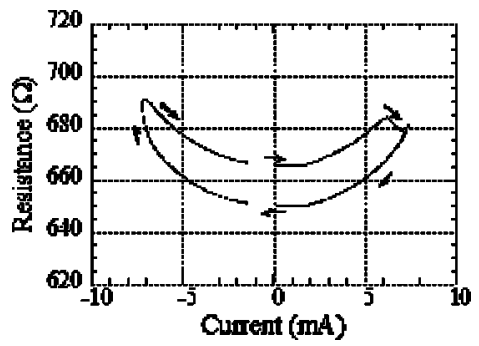

FIG. 4. Resistance as a function of current in a MnIr/CoFe/Cu/CoFe $/ \mathrm{NiFe}$ Nanopillar measured using Pt- coated probe. Arrows show current sweep direction during measurement. 


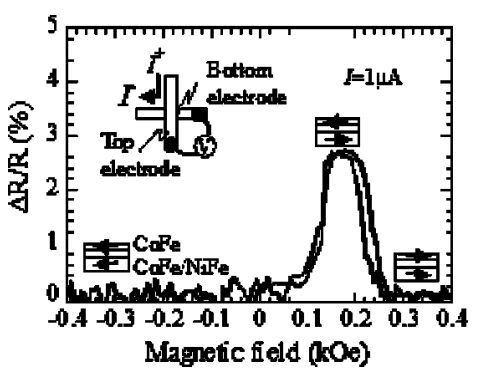

FIG. 5. Resistance as a function of magnetic field in $\mathrm{MnIr} / \mathrm{CoFe} / \mathrm{Cu} / \mathrm{CoFe} / \mathrm{NiFe}$ nanopillar using the four-probe method. Schematic shows geometry of current during measurement.

As the current is increased, there is a symmetric gradual rise in resistance with respect to the current direction, as shown in Fig. 4. When we applied a current of $10 \mathrm{~mA}$, the point of the PtIr-coated probe started to melt, as shown in the

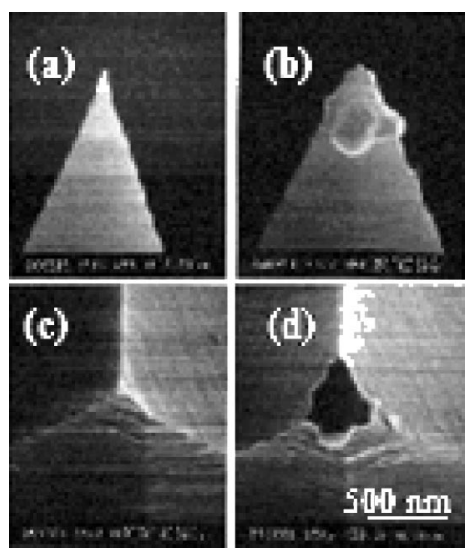

FIG. 6. SEM images of the point of a PtIr-coated cantilever; $(a, c)$ initial image and (b,d) images after $10 \mathrm{~mA}$ current was applied. (a) and (b) show side view, and (b) and (d) show top view.
SEM images in Fig. 6. Because the probe point had an area of less than $50 \mathrm{~nm}$, application of a $10 \mathrm{~mA}$ current resulted in a current density of $1 \times 10^{9} \mathrm{~A} / \mathrm{cm}^{2}$. An $I-V$ curve measured using the melted probe exhibited a nonlinear response, suggesting that the Si surface of the cantilever was exposed.

\section{CONCLUSION}

In conclusion, we have observed current-driven hysteretic switching using AFM probe contact in an exchange biased spin-valve $\mathrm{MnIr} / \mathrm{CoFe} / \mathrm{Cu} / \mathrm{CoFe} / \mathrm{NiFe}$ nanopillar. This probe contact technique, incorporating current-driven switching, is promising for production of magnetic memory devices with ultrahigh areal densities.

\section{ACKNOWLEDGMENTS}

This work was supported by the IT-program of Research Revolution 2002 (RR2002). We are grateful to Masayoshi Ishibashi and Mieko Ishii of Hitachi Advanced Research Laboratory for their technical help, and to the EPEL staff for their helpful discussions and invaluable support.

${ }^{1}$ J. Slonczewski, J. Magn. Magn. Mater. 159, L1 (1996).

${ }^{2}$ L. Berger, Phys. Rev. B 54, 9353 (1996).

${ }^{3}$ M. Tsoi, A. G. M. Jansen, J. Bass, W. C. Chiang, M. Seck, V. Tsoi, and P. Wyder, Phys. Rev. Lett. 80, 4281 (1998).

${ }^{4}$ E. B. Myers, D. C. Ralph, J. A. Katine, R. N. Louie, and R. A. Buhrman, Science 285, 867 (1999).

${ }^{5}$ J. A. Katine, F. J. Albert, R. A. Buhrman, E. B. Myers, and D. C. Ralph, Phys. Rev. Lett. 84, 3149 (2000).

${ }^{6}$ F. J. Albert, J. A. Katine, R. A. Buhman, and D. C. Ralph, Appl. Phys. Lett. 77, 3809 (2000).

7J. Grollier et al., Appl. Phys. Lett. 78, 3663 (2001).

${ }^{8}$ J. Grollier et al., Phys. Rev. B 67, 174402 (2003).

${ }^{9}$ J.-E. Wegrowe, X. Hoffer, Ph. Guittienne, A. Fabian, L. Gravier, T. Wade, and J.-Ph. Ansermet, J. Appl. Phys. 91, 6806 (2002).

${ }^{10}$ S. Urazhdin, H. Kurt, W. P. Pratt, Jr., and J. Bass, Appl. Phys. Lett. 83, 114 (2003)

${ }^{11}$ P. Vettiger et al., IEEE Trans. Nanotechnol. 1, 39 (2002). 